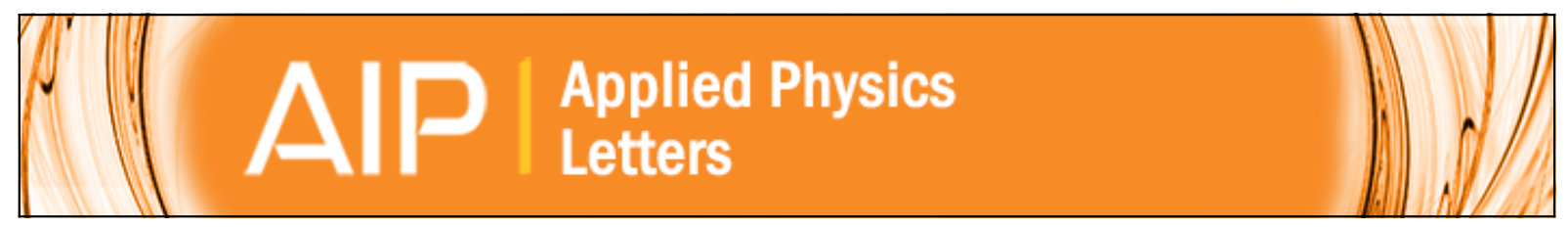

\title{
Collinear second harmonic generations in a nonlinear photonic quasicrystal
}

Yan Sheng, Kaloian Koynov, Junhong Dou, Boqin Ma, Jingjuan Li, and Daozhong Zhang

Citation: Applied Physics Letters 92, 201113 (2008); doi: 10.1063/1.2937210

View online: http://dx.doi.org/10.1063/1.2937210

View Table of Contents: http://scitation.aip.org/content/aip/journal/apl/92/20?ver=pdfcov

Published by the AIP Publishing

\section{Articles you may be interested in}

Second-harmonic generation in a periodically poled congruent $\mathrm{LiTaO} 3$ sample with phase-tuned nonlinear Cherenkov radiation

Appl. Phys. Lett. 100, 022905 (2012); 10.1063/1.3676440

All-optical polarization switch in a quadratic nonlinear photonic quasicrystal

Appl. Phys. Lett. 94, 091108 (2009); 10.1063/1.3090488

Broadband quasi-phase-matched second-harmonic generation in a nonlinear photonic crystal

J. Appl. Phys. 100, 023120 (2006); 10.1063/1.2213150

Quasiphase matched harmonic generation in a two-dimensional octagonal photonic superlattice Appl. Phys. Lett. 87, 251103 (2005); 10.1063/1.2138352

Second-harmonic green generation from two-dimensional (2) nonlinear photonic crystal with orthorhombic lattice structure

Appl. Phys. Lett. 83, 3447 (2003); 10.1063/1.1622786

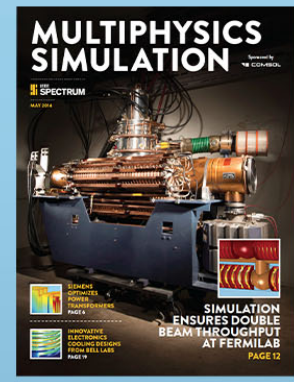




\title{
Collinear second harmonic generations in a nonlinear photonic quasicrystal
}

\author{
Yan Sheng, ${ }^{1, a)}$ Kaloian Koynov, ${ }^{1}$ Junhong Dou, ${ }^{2}$ Boqin $\mathrm{Ma}^{2}{ }^{2}$ Jingjuan $\mathrm{Li}^{2}{ }^{2}$ and \\ Daozhong Zhang ${ }^{2}$ \\ ${ }_{1}^{1}$ Max Plank Institute for Polymer Research, Ackermannweg 10, D-55128 Mainz, Germany \\ ${ }^{2}$ Optical Physics Lab., Beijing National Laboratory for Condensed Matter Physics, Institute of Physics, \\ Chinese Academy of Sciences, Beijing 100080, People's Republic of China
}

(Received 23 March 2008; accepted 7 May 2008; published online 23 May 2008)

\begin{abstract}
$\mathrm{LiNbO}_{3}$ nonlinear photonic quasicrystal with octagonal symmetry was fabricated. The collinear quasiphase matched second harmonic generations at 22 wavelengths were observed. It is shown that not only the standard type quasiphase matching but also the recently proposed projection-based quasiphase matching plays an important role in these harmonic generations. (C) 2008 American Institute of Physics. [DOI: 10.1063/1.2937210]
\end{abstract}

Quasiphase matching (QPM) is a well-established technique for improving the efficiencies of nonlinear optical interactions by applying a periodic variation to the sign of the second order nonlinear coefficient $\chi^{(2)}{ }^{1}$ Such periodical structures, also known as nonlinear photonic crystals (NPCs), exhibit a set of reciprocal vectors (RVs) that may help to satisfy momentum conservation for a given nonlinear optical process. While initially one-dimensional (1D) structures were considered, in the last decades, two-dimensional (2D) NPC were suggested ${ }^{2,3}$ and extensively studied. ${ }^{4-7}$ Even more recently, the so called nonlinear photonic quasicrystals (NPQCs) having a quasiperiodic 2D spatial distribution of $\chi^{(2)}$ attracted much attention. Such 2D quasiperiodic structures have a greater density of RVs than their periodic counterparts, and therefore, can provide a superior flexibility in achieving QPM. ${ }^{8-12}$

It is generally accepted that efficient QPM second harmonic generation (SHG) requires the existence of a RV equal to the phase-mismatch vector of the interacting beams, i.e.,

$$
\vec{G}=\vec{k}_{2}-2 \vec{k}_{1},
$$

where $\vec{G}$ is $\mathrm{RV}$ and $\vec{k}_{1}$ and $\vec{k}_{2}$ are the wave vectors of the fundamental and the second harmonic beams, respectively. Very recently, Bahabad et al. have proposed the so called projection-based QPM, demonstrating that when using finite width beams for collinear interactions within a periodic structure, momentum conservation can also be satisfied using a projection of the RV onto the direction of propagation. ${ }^{13}$

In this letter, we show that projection-based QPM can be achieved not only in periodic structures but also in NPQCs. Moreover, using the dense nature of the RVs of the latter, we observed experimentally a collinear SHG of at least 22 wavelengths using a single NPQC. In addition, our experiments performed with a Gaussian pump beam with a waist diameter of $100 \mu \mathrm{m}$ demonstrate that there is no rigorous requirement for a finite interaction width when the projection-based QPM is applied to NPQC.

The NPQC used was a 2D octagonal quasiperiodic superlattice, fabricated by electrical field poling of a $\mathrm{z}$-cut $\mathrm{LiNbO}_{3}$ wafer. ${ }^{14}$ The length, width, and thickness of the sample were $8.5,8.5$, and $0.4 \mathrm{~mm}$ respectively. An optical micrograph of the $+\mathrm{z}$ surface of the wafer after acid etching is shown in Fig. 1. The octagonal quasiperiodic geometry,

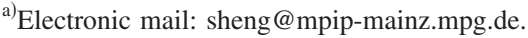

clearly visible on the figure, was obtained by tiling the plane with two kinds of tiles: squares and $45^{\circ}$ rhombi. The side length of the squares and rhombi was $a=11.5 \mu \mathrm{m}$. The poled domains were placed at the vertexes of the square and rhombus and their diameter was $d=4.6 \mu \mathrm{m}$.

Figure 2(a) shows the diffraction pattern of the NQPC oriented with its eightfold axis parallel to the incident $\mathrm{He}-\mathrm{Ne}$ laser beam. The figure actually resembles the Fourier transform of the real space lattice. The locations of the diffractive spots correspond to the locations of RVs that can be used for QPM, and the size of the spots indicates the size of the corresponding Fourier coefficients. No simple periodicity can be derived from the picture, but it is evident that the diffractive spots very often form a configuration of octagons or squares with different sizes. Such patterns agree well with the octagonal quasiperiodic tiling in the real space. In order to index the RVs, we often use four basic reciprocal vectors $\vec{F}_{i}(i=1,2,3,4)$, as shown in Fig. 2(a). Such representation, however, becomes heavy and difficult to apply when a large number of RVs need indexing. As shown below, more than $20 \mathrm{RVs}$ need to be addressed in this work, and therefore, for simplicity, we choose to use only one integer number to index the RVs. Figure 2(b) gives an expanded view of the reciprocal space within a $2 \pi / 8$ sector, where the RVs to be used later are marked and $x$ axis is defined. As can be seen in this representation, RV8, for example, corresponds to the unit vector $\vec{F}_{3}$, RV3 to $\vec{F}_{4}$, or RV19 to $\vec{F}_{3}+\vec{F}_{4}$. Furthermore, Fig. 2 shows that at least eleven reciprocal vectors $(1,2,4,5$, $8,11,12,15,20,21,22)$ are oriented along the $x$ axis, and therefore, the QPM conditions for collinear SHG should be satisfied for a number of laser wavelengths. The magnitudes of all RVs described above were measured by comparing the diffraction pattern shown on Fig. 2 with that of a 1D grating

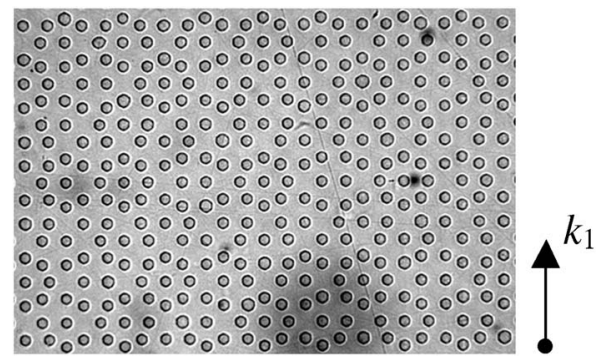

FIG. 1. Micrograph of the poled NPQC. The arrow indicates the fundamental beam propagation direction. 


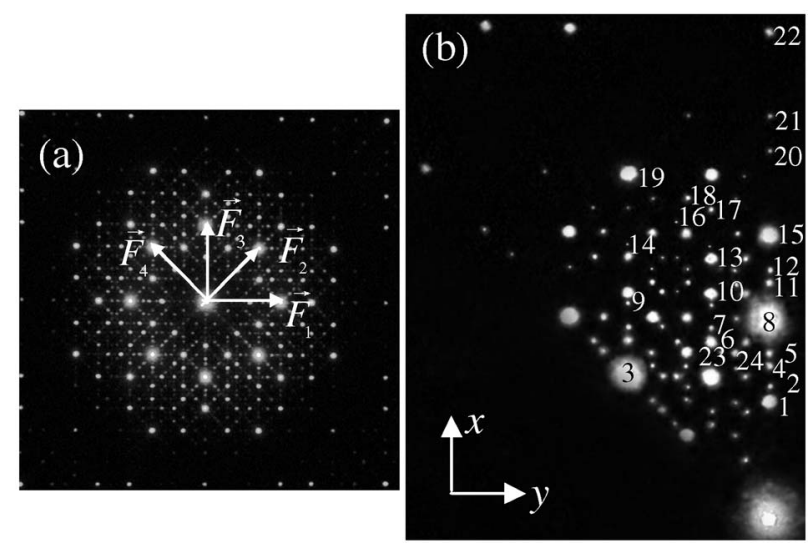

FIG. 2. (a) The diffraction pattern of $\mathrm{LiNbO}_{3} \mathrm{NPQC}$. (b) An expanded view on the diffraction pattern within a $2 \pi / 8$ sector.

with known period. Furthermore, the value found for the basic vectors $\left|F_{i}\right|=0.632 \mu \mathrm{m}^{-1}$ was related to the tile length using the empirical approximation $\left|\vec{F}_{i}\right|=4 \pi /(\sqrt{3} a)$ as in earlier work. ${ }^{10}$

The collinear SHG experiments were performed at $24.5^{\circ} \mathrm{C}$ using a nanosecond optical parametric oscillator (OPO) pumped by a Nd:yttrium aluminum garnet laser. The OPO produces laser pulses with a duration of $4 \mathrm{~ns}$ at a repetition rate of $10 \mathrm{~Hz}$. The laser beam was entering along the $x$ axis and focused on the entrance surface of the sample by a $10 \mathrm{~cm}$ focal-length lens that produced a focal spot with a beam waist of $\sim 100 \mu \mathrm{m}$ inside the crystal. A fiber optic spectrometer was used to measure the wavelengths of fundamental and harmonic beams.

Using this setup, we were able to observe collinear SHG for at least 22 different fundamental wavelengths in the range from 0.915 to $1.482 \mu \mathrm{m}$, as schematically shown on Fig. 3. The fundamental wavelengths are also listed in Table I together with the SHG efficiencies, the respective phasemismatch vectors, and the corresponding RVs that help to

TABLE I. Collinear SHGs in the NPQC. The wavelength $(\lambda)$ is in unit of $\mu \mathrm{m}$ and $\mathrm{RV}$ is in unit of $\mu \mathrm{m}^{-1}$. Sellmeier coefficients at $24.5^{\circ} \mathrm{C}$ were used to calculate $\Delta k^{15}$

\begin{tabular}{|c|c|c|c|c|c|c|}
\hline \multicolumn{2}{|c|}{ Experiment } & \multirow[b]{2}{*}{ No. } & \multicolumn{2}{|c|}{ Theory } & \multirow{2}{*}{$\begin{array}{c}\text { Efficiency } \\
(\%)\end{array}$} & \multirow[b]{2}{*}{$(m, n)$} \\
\hline$\lambda$ & $\Delta k$ & & RV & projection & & \\
\hline 1.482 & 0.367 & 1 & 0.370 & & 9.0 & $(-2,2)$ \\
\hline 1.429 & 0.401 & 2 & 0.402 & & 1.5 & $(-9,7)$ \\
\hline 1.375 & 0.442 & 3 & & 0.447 & 18.0 & $(1,0)$ \\
\hline 1.346 & 0.468 & 4 & 0.479 & & 1.8 & $(-6,5)$ \\
\hline 1.318 & 0.495 & 5 & 0.492 & & 1.8 & $(11,-7)$ \\
\hline 1.270 & 0.548 & 6 & & 0.555 & 2.5 & $(-3,3)$ \\
\hline 1.226 & 0.605 & 7 & & 0.600 & 1.5 & $(7,-4)$ \\
\hline 1.207 & 0.633 & 8 & 0.632 & & 36.0 & $(0,1)$ \\
\hline 1.190 & 0.660 & 9 & & 0.664 & 1.3 & $(-7,6)$ \\
\hline 1.157 & 0.717 & 10 & & 0.709 & 3.6 & $(3,-1)$ \\
\hline 1.131 & 0.767 & 11 & 0.772 & & 1.0 & $(-11,9)$ \\
\hline 1.117 & 0.797 & 12 & 0.785 & & 1.2 & $(6,-3)$ \\
\hline 1.106 & 0.821 & 13 & & 0.817 & 1.8 & $(-1,2)$ \\
\hline 1.086 & 0.869 & 14 & & 0.862 & 0.8 & $(9,-5)$ \\
\hline 1.075 & 0.897 & 15 & 0.894 & & 20.0 & $(2,0)$ \\
\hline 1.066 & 0.921 & 16 & & 0.926 & 0.4 & $(-5,5)$ \\
\hline 1.047 & 0.974 & 17 & & 0.970 & 0.4 & $(5,-2)$ \\
\hline 1.039 & 0.998 & 18 & & 1.002 & 0.7 & $(-2,3)$ \\
\hline 1.012 & 1.086 & 19 & & 1.079 & 1.3 & $(1,1)$ \\
\hline 0.991 & 1.163 & 20 & 1.156 & & 0.5 & $(4,-1)$ \\
\hline 0.965 & 1.269 & 21 & 1.264 & & 0.4 & $(0,2)$ \\
\hline 0.915 & 1.517 & 22 & 1.526 & 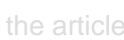 & 56 & $(2,1)$ \\
\hline
\end{tabular}

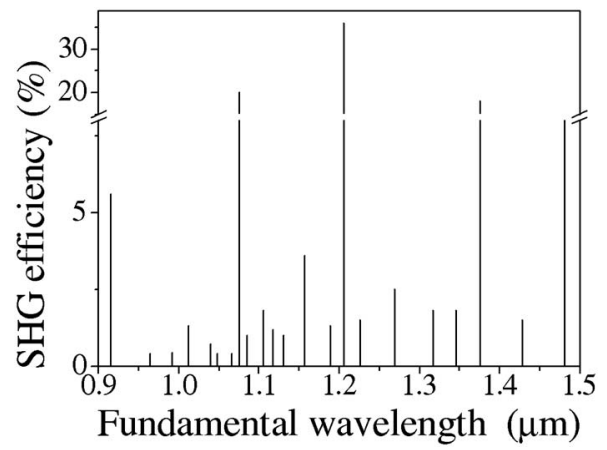

FIG. 3. Schematic representation of the collinear SHGs observed in the octagonal NPQC.

satisfy the QPM conditions as discussed below. Clearly, the SHG efficiency depends strongly on the wavelength. There are five obvious harmonic peeks, at which the efficiencies are much higher than those of others. As shown later, this is ascribed to the larger Fourier coefficients of the corresponding RVs. While the efficiencies were significantly lower for the rest of the wavelengths, in all cases, clear and reproducible SHG was observed.

Such collinear SHG observed at as many as 22 wavelengths can not be explained with standard QPM only and needs more thoughtful interpretation. Let us first consider the $\mathrm{RV}$ oriented along the fundamental wave vector, which can help to directly satisfy the phase matching conditions for a collinear SHG. For an octagonal NPQC with tile side length of $11.5 \mu \mathrm{m}$, the strongest three RVs along $x$ axis (see Fig. 2) are $\mathrm{RV} 1=0.370 \mu \mathrm{m}^{-1}, \quad \mathrm{RV} 8=0.632 \mu \mathrm{m}^{-1}, \quad$ and $\mathrm{RV} 15$ $=0.894 \mu \mathrm{m}^{-1}$. These three RV correspond to the strong SHG observed at fundamental wavelengths of $1.482,1.207$, and $1.075 \mu \mathrm{m}$, respectively. The resultant SHG efficiencies were $9 \%, 36 \%$, and $20 \%$ for the input fundamental power of $3.6 \mathrm{~mW}$. As can be seen in Fig. 2, in addition to these three strong RVs, further weaker RVs $(2,4,5,11,12,20,21,22)$ are also oriented along the $x$ axis. As a result, the collinear SHG can be achieved at additional wavelengths. The corresponding SHG efficiencies (Fig. 3, Table I) are significantly lower than those discussed above, because of the smaller Fourier coefficients of these RVs.

In this way, using the standard QPM theory, i.e., Eq. (1), we can explain the observed collinear SHG at eleven wavelengths. As shown in Table I, for each of these wavelengths, a reciprocal vector exist, which is oriented along the beam propagation axes and has an amplitude equal to the corresponding phase-mismatch $\vec{k}_{2 \omega}-2 \vec{k}_{\omega}$. Such considerations, however, can not explain the SHG at the rest of the wavelengths shown in Fig. 3 and in Table I. For example, SHG were observed at fundamental wavelengths of 1.375 and $1.106 \mu \mathrm{m}$, for which RVs equal to 0.442 and $0.821 \mu \mathrm{m}^{-1}$ are, respectively, required to maintain monument conservation. ${ }^{15}$ However, as shown in Fig. 4, no such RVs can be found in the reciprocal space. Motivated by the very recent work of Bahabad et al. ${ }^{13}$ we project some of the existing RVs onto the beam propagation direction ( $x$ axis). As can be seen in Fig. 4, the projection "arms" of RV3 and RV13 (0.447 and $0.817 \mu \mathrm{m}^{-1}$ ) fit very well to the corresponding phase-mismatch values discussed above. This suggests that the projection-based QPM may play a key role in the observed SHGs. We have extended this analysis for all wavelengths at which collinear SHG was observed. As can 


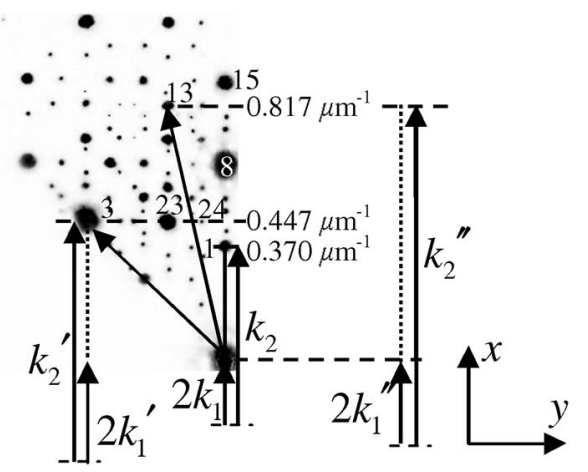

FIG. 4. The QPM schemes in reciprocal vector space. The momentum conservation between $k_{2}$ and $2 k_{1}$ is achieved using standard QPM and those for $k_{2}^{\prime}$ and $k_{2}^{\prime \prime}$ are projection-based. The real lines represent the wave vectors and $\mathrm{RV}$, and the dashed lines denote projections of RVs.

be seen from Table I, in all cases, we were able to find a RV at which projection on to the beam propagation direction satisfies the QPM conditions. It should be emphasized at this point that the reported effect is an experimental observation for which we do not have a detailed theoretical explanation. Nevertheless, the usage of finite width beams is probably the main reason for the projection-based QPM interactions in NPQC, in a way similar to that reported for periodic NPC. ${ }^{13}$ Two important phenomena related to the projection-based QPM in NPQC deserve a special discussion. First of all, the projections of the RV with larger Fourier coefficient ensure higher SHG efficiency. For example the measured efficiency was $\sim 18 \%$ at fundamental wavelength $1.375 \mu \mathrm{m}$, for which the projection of the very strong RV3 satisfies the momentum conversation, while only $1.8 \%$ at $\lambda=1.106 \mu \mathrm{m}$, where the projection of the weaker RV13, was used. Second, as shown in Fig. 4, there are many RVs that share the same projection arms, such as the projections of RV3, 23, 24, etc., are all equal to $0.447 \mu \mathrm{m}^{-1}$. In the analysis above, we considered only the strongest one among them, i.e., RV3. Most likely, however, all of these projections sum up in their contribution to the SHG efficiency.

It is important to emphasize at this point that the standard QPM and the projection-based QPM can be described in one joint approach. Indeed, let us consider again the four basic reciprocal vectors $\vec{F}_{i}$ shown in Fig. 2(a). As described above, they have equal amplitudes $\left|\vec{F}_{i}\right|=4 \pi /(\sqrt{3} a)=F$. The projections of these basic vectors along the $x$ axis are 0 , $F / \sqrt{2}, F$, and $F / \sqrt{2}$, respectively. It is clear, therefore, that there are two basic vectors with different amplitudes, i.e., $F / \sqrt{2}$ and $F$, along the $x$ axis, and the QPM collinear SHG can be satisfied from a generalized reciprocal vector that has the form

$$
G_{m, n}=(m / \sqrt{2}+n) F,
$$

where $(m, n)$ is any pair of integer numbers. As shown in the last column of Table I, we were able to find such pairs of $m$ and $n$ for all 22 fundamental wavelengths at which collinear SHG was observed. Not surprisingly, the SHG efficiency decreases when the values of $m$ and $n$ increase, revealing a decrease in the corresponding Fourier coefficients. In the same time, it should be pointed out that for some values of $m$ and $n$, no SHG was detected. Similar effects exist in periodic or Fibonacci quasiperiodic structures, ${ }^{16}$ for which the strong dependence between the SHG efficiency and the reversed duty cycle plays a key role. In 1D periodic structures, for example, the SHG efficiency is proportional to $(2 / j \pi) \sin (j \pi D)$, where $j$ is the order of QPM and $D$ is the reversed duty cycle. A reversed duty cycle of $50 \%$ can destroy completely the second-order $(j=2)$ QPM. Similar considerations may explain the results observed in the 2D NQPC as well.

In conclusion, we have fabricated a $\mathrm{LiNbO}_{3} \mathrm{NPQC}$ with octagonal symmetry. Using this NPQC, we observed experimentally collinear SHG at 22 fundamental wavelengths in the range from 0.915 to $1.482 \mu \mathrm{m}$. This very large number of wavelengths cannot be explained using the conventional QPM approach only and clearly shows that momentum conservation can also be satisfied using a projection of the RV onto the propagation direction of the beams.

The financial support of Chinese Academy of Science, Max Plank Society, and Deutscher Akademischer Austauschdienst (DAAD) is gratefully acknowledged.

${ }^{1}$ M. Fejer, G. Magel, D. Jundt, and R. Byer, IEEE J. Quantum Electron. 28, 2631 (1992).

${ }^{2}$ V. Berger, Phys. Rev. Lett. 81, 4136 (1998).

${ }^{3}$ N. G. R. Broderick, G. W. Ross, H. L. Offerhaus, D. J. Richardson, and D. C. Hanna, Phys. Rev. Lett. 84, 4345 (2000).

${ }^{4}$ S. M. Saltiel, D. N. Neshev, R. Fischer, W. Krolikowski, A. Arie, and Y. S. Kivshar, Phys. Rev. Lett. 100, 103902 (2008).

${ }^{5}$ N. G. R. Broderick, R. T. Bratfalean, T. M. Monro, D. J. Richardson, and C. M. de Sterke, J. Opt. Soc. Am. B 19, 2263 (2002).

${ }^{6}$ N. Fujioka, S. Ashihara, H. Ono, T. Shimura, and K. Kuroda, J. Opt. Soc. Am. B 24, 2394 (2007).

${ }^{7}$ Y. Sheng, J. Dou, B. Ma, B. Cheng, and D. Zhang, Appl. Phys. Lett. 91, 011101 (2007).

${ }^{8}$ R. T. Bratfalean, A. C. Peacock, N. G. R. Broderick, and K. Gallo, Opt. Lett. 30, 424 (2005).

${ }^{9}$ K. Fradkin-Kashi, A. Arie, P. Urenski, and G. Rosenman, Phys. Rev. Lett. 95, 133901 (2005).

${ }^{10}$ B. Ma, T. Wang, Y. Sheng, P. Ni, Y. Wang, B. Cheng, and D. Zhang, Appl. Phys. Lett. 87, 251103 (2005).

${ }^{11}$ Y. Sheng, J. Dou, B. Cheng, and D. Zhang, Appl. Phys. B: Lasers Opt. 87, 603 (2007).

${ }^{12}$ B. Ma, Y. Sheng, J. Dou, B. Cheng, and D. Zhang, Opt. Commun. 274, 429 (2007).

${ }^{13}$ A. Bahabad, N. Voloch, A. Arie, A. Bruner, and D. Eger, Phys. Rev. Lett. 98, 205501 (2007).

${ }^{14}$ Y. Sheng, T. Wang, B. Ma, B. Cheng, and D. Zhang, Appl. Phys. Lett. 88, 041121 (2006).

${ }^{15}$ D. H. Jundt, Opt. Lett. 22, 1553 (1997).

${ }^{16}$ S. Zhu, Y. Zhu, Y. Qin, H. Wang, C. Ge, and N. Ming, Phys. Rev. Lett. 78, 2752 (1997). 